\title{
ANÁLISE COMPARATIVA DOS MODOS E FREQUENCIAS NATURAIS DE VIBRAÇÃO DE VIGAS ENGASTADA LIVRE E BI- ENGASTADA SUJEITAS À OSCILAÇÕES LIVRES
}

\section{COMPARATIVE ANALYSIS OF THE MANNERS AND NATURAL FREQUENCIES OF VIBRATION OF BEAMS CLAMPED-FREE AND CLAMPED-CLAMPED SUBJECT TO FREE OSCILLATIONS}

\section{Cássio Fabian Sarquis de Campos ${ }^{1}$; Daniele Araújo Altran ${ }^{2}$; Gustavo Figueiredo Formagio}

\author{
${ }^{1}$ Universidade do Oeste Paulista, Faculdade de engenharia, Pres. Prudente, SP \\ ${ }^{2}$ Universidade Federal da Grande Dourados, Faculdade de engenharia - FAEN, \\ Dourados, MS
}

RESUMO - O estudo da dinâmica de estruturas rígidas ou flexíveis sob a ação de forças externas, tais como, ondas regulares marítimas, ventos, vibrações mecânicas externas de qualquer ordem, é de extrema importância para a prevenção de possíveis problemas estruturais, sendo, por exemplo, trincas, deslocamentos, rupturas parciais ou totais dessas estruturas. Este trabalho teve como objetivo realizar a modelagem teórica e computacional de duas vigas idênticas sendo uma do tipo engastada livre e a outra bi engastada, de modo a obter a exibir representações gráficas de seus quatro primeiros modos naturais de vibrações e suas respectivas frequências naturais. $O$ estudo realizado foi mediado pela modelagem computacional dos modos naturais de vibração para cada viga. A modelagem teórica do comportamento de cada viga ao longo de seu comprimento bem como os valores das frequências naturais de vibração seguiu o método dos modos assumidos. O recurso computacional utilizado para exibir os modos de vibração ao longo do comprimento de cada viga foi o software Matlab ${ }^{\circledR}$. Este trabalho proporcionou o conhecimento matemático de modelagem dinâmica de estruturas com vínculos diferentes. Observou-se que as frequências naturais da viga bi engastada para os quatro modos de vibração foram maiores que as frequências da viga engastada livre devido sua limitação da vibração pelo segundo engaste.

Palavras-chave: dinâmica; vibração; viga; engastada livre; bi engastada.

Recebido em: $10 / 08 / 2018$ Revisado em: 28/08/2018 Aprovado em: 20/09/2018
ABSTRACT - The objective of this work was the theoretical and computational modeling of free-form and bi-set beams, objectively showing their ways of vibrations and their natural frequencies. The study of the dynamics of rigid or flexible structures, under the action of external forces, such as regular marine waves, winds, external mechanical vibrations of any order, is of extreme importance for the prevention of possible structural problems, for example, displacements, partial or total ruptures of these structures. All the study was carried out 
using the methods of Computational Analysis of the Natural Vibration Modes and Frequencies of a Free Beam of Forms for Free Frequencies and Computational Calculation of Lies and Natural Vibration Frequencies of a Beam Without Free Sets of Free Oscillations and the Computational Computation of Lies and Natural Frequencies of Vibration of a Bi Beam established by the Method of Assumed Modalities. The computational resources used to do the theoretical remodeling of the beams and their functions were Matlab ${ }^{\circledR}$ and Maple ${ }^{\circledR}$. Employees to implement three-dimensional vibration modes and development of new studies with different bundles and physical properties. This comparative work of the beams provided the mathematical and dynamic knowledge of modeling of the structures, being they, modeling skills through programming.

Keywords: dynamics; vibration; beam; crimped-free; crimped-crimped. 


\section{INTRODUÇÃO}

A Engenharia Estrutural é uma área existencial da Engenharia Civil, definida como a área onde o conhecimento do comportamento de estruturas estáticas e dinâmicas são de extrema importância. Isto para otimizar projetos e dimensionar os mesmos, pois, de forma bem abrangente, as estruturas são fabricadas por meio de técnicas e elementos estruturais onde suas cargas solicitantes são transmitidas desde pontos mais elevados de sua edificação até pontos mais periféricos, como suas fundações. Entre os elementos geralmente utilizados, tem-se um destaque as lajes, vigas e pilares. (ARAUJO et al., 2004).

O objetivo do projeto de uma estrutura é permitir que a mesma atenda à sua função primária sem entrar em colapso e sem deformar ou vibrar excessivamente. Dentro destes limites, os quais são precisamente definidos por normas técnicas, o engenheiro estrutural tem como objetivo e função obter o melhor uso dos materiais disponíveis o menor custo possível de construção e manutenção da estrutura.

Resumidamente, as principais etapas do projeto estrutural podem ser citados como segue, a criação do esquema estrutural, a definição das cargas ou forças que atuam na estrutura, o cálculo dos esforços e deformações, o dimensionamento das peças estruturais, e finalmente $o$ detalhamento do projeto para execução.

O estudo do comportamento das vigas é um fundamento importante na engenharia estrutural por visar sua função as respostas estáticas e dinâmicas dos componentes estruturais sob a ação de forças externas aplicadas em seu módulo estrutural de modo a estudar e obter informações de análises sobre o comportamento desta estrutura em situações complexas próximas da realidade.

Neste contexto, a análise estrutural dinâmica tem por finalidade quantificar os esforços internos, velocidades, acelerações e os deslocamentos que são desenvolvidos em qualquer sistema estrutural quando o mesmo é submetido a um carregamento arbitrário, cuja intensidade, direção e sentido variem com o tempo. (RODRIGUES, 1997).

$\mathrm{Na}$ dinâmica das estruturas, uma grande preocupação, é ocorrência de vibrações excessivas, indesejáveis do ponto de vista estrutural, já que podem causar danos na estrutura devido ao alto valor dos esforços solicitantes provocados. As vibrações também podem ocasionar a fadiga dos elementos estruturais, além de provocar desconforto nos indivíduos que utilizam a estrutura regularmente, além de, comprometer sua segurança.

( , 2007).

Desse modo, desde a disseminação da informática ocorrida no final da década de 1980, foram promovidos muitos avanços tecnológicos, com grande aumento da capacidade de armazenamento, gerenciamento e processamento de dados apresentados pelos computadores de pequeno porte.

O Engenheiro de estrutural passou a ter acesso a equipamentos e programas computacionais que possibilitam análises baseada em modelos de estruturas, proporcionando um aumento da confiabilidade e diminuição de custos dos projetos e construções (WAIDEMAN, 2004, p.1).

\section{OBJETIVO}

O objetivo deste trabalho foi de realizar a modelagem teórica e computacional de duas vigas idênticas com tipos de engastes distintos, sendo uma do tipo engastada livre e a outra bi engastada.

Obter e representar graficamente os quatro primeiros modos de vibrações para todos os casos propostos, e encontrar os valores dessas frequências de vibração também constitui objetivo deste trabalho. 


\section{METODOLOGIA}

O estudo se iniciou a partir das descrições do comportamento de vigas propostas nos artigos de iniciação cientifica publicados na revista Colloquium Exactarum com os temas Análise Computacional dos Modos e Frequências Naturais de Vibração de uma Viga Engastada-Livre Sujeita a Oscilações Livres $(M L E)$ e Cálculo Computacional dos Modos e das Frequências Naturais de Vibração de uma Viga Bi engastadas pelo Método dos Modos Assumidos (MBE).

As vigas agora serão comparadas de modo que suas dimensões e propriedades físicas são idênticas e as condições de contorno para cada tipo de engaste foi respeitadas.

Limitou-se as equações de movimento das vigas no plano $x y$, de modo que ao longo do eixo $x$ temos o comprimento da viga e no eixo y a amplitude da vibração.

Utilizando o método dos modos assumidos, matematicamente, pode-se escrever a solução dos movimentos flexíveis a partir da seguinte equação (Junkins e Kim, 1993).

$$
y_{i}(x ; t)=\sum_{i=1}^{n} \Phi_{i}(x) \Psi_{i}(t)
$$

Onde, $i$ corresponde ao modo de vibração, $\Psi_{i}(t)$ corresponde a função do movimento dependente do tempo e $\Phi_{i}(x)$ denota as funções de forma escolhidas para descrever as deflexões das vigas ao longo de seu comprimento (MEIROVITCH, 1990).

Benhard (1943) menciona que as funções assumidas apresentam condições de contornos específicos de acordo com o tipo de vínculo das vigas.

Young e Felgar (1949) apresentam as formas das funções que serão assumidas, neste estudo, como representantes da parte flexível das estruturas. Estas funções são dadas por:

$$
\begin{gathered}
\Phi_{i}(x)=\cosh \left(\beta_{i} \cdot x\right)-\cos \left(\beta_{i} \cdot x\right)- \\
\alpha_{n}\left[\operatorname{senh}\left(\beta_{i} \cdot x\right)-\operatorname{sen}\left(\beta_{i} \cdot x\right)\right]
\end{gathered}
$$

Onde, $x$ se refere ao comprimento das vigas de 0 à $L, \beta$ sintetiza constantes relacionada a frequência natural de vibração e $\alpha_{n}$ corresponde a valores característicos do tipo de viga no sistema de engaste.

Estes valores de $\alpha$ e $\beta$ são específicos quanto ao tipo de engaste da viga e foram obtidos da "Tables of Characteristic Functions Representing Normal Modes of Vibration of a Beam" de Dana Young e Robert, P. Felgar Jr (1949) sendo apresentados nas tabelas 1 e 2 a seguir.

Tabela 1. Valores de $\alpha$ e e $\beta$.

Fonte: (Young e Felgar, 1949).

\begin{tabular}{c|c|c}
\cline { 2 - 3 } & \multicolumn{2}{|c}{ Engastada Livre } \\
\hline Modo $(\boldsymbol{n})$ & $\boldsymbol{\beta}$ & $\boldsymbol{\alpha}_{\boldsymbol{n}}$ \\
\hline $\mathbf{1}^{\circ}$ & 1,9751041 & 0,734955 \\
\hline $\mathbf{2}^{\circ}$ & 4,68409113 & 1,01846644 \\
\hline $\mathbf{3}^{\circ}$ & 7,85475743 & 0,99922450 \\
\hline $\mathbf{4}^{\circ}$ & 10,99554074 & 1,000033553 \\
\hline
\end{tabular}


Tabela 2. Valores de $\alpha$ e e $\beta$.

Fonte: (Young e Felgar, 1949).

\begin{tabular}{c|c|c}
\cline { 2 - 3 } & \multicolumn{2}{|c}{ Bi Engastada } \\
\hline Modo $(\boldsymbol{n})$ & $\boldsymbol{\beta}$ & $\boldsymbol{\alpha}_{\boldsymbol{n}}$ \\
\hline $\mathbf{1}^{\circ}$ & 4,7300408 & 0,9825022158 \\
\hline $\mathbf{2}^{\circ}$ & 7,8532046 & 1,000777311 \\
\hline $\mathbf{3}^{\circ}$ & 10,9956058 & 0,9999664501 \\
\hline $\mathbf{4}^{\circ}$ & 14,1371655 & 1,000001450 \\
\hline
\end{tabular}

Segundo Thomson, (1981) para condições de contorno da viga bi engastada são de que o deslocamento $\emptyset_{i}(x)=0 \mathrm{e}$ $\emptyset_{i}(L)=0$ assegurando que não haja vibração em tais pontos garantindo assim que na implementação computacional a função apresentada anteriormente satisfaz estas condições de contorno geométricas.

Para modelar as vigas elegeu-se as seguintes características físicas, supondo que o material das vigas é de concreto com seção quadrada (Tabela 3).

Tabela 3. Especificações das vigas.

\begin{tabular}{c|c|c}
\hline Propriedade & Símbolo & Intensidade \\
\hline Massa específica volumétrica do concreto & $\boldsymbol{\rho}$ & $2800 \mathrm{Kg} / \mathrm{m}^{3}$ \\
\hline Massa da viga & $\boldsymbol{m}$ & $63,0 \mathrm{Kg}$ \\
\hline Área de seção & $\boldsymbol{A}$ & $0,15 \mathrm{~m} \mathrm{X0,15} \mathrm{\textrm {m }}$ \\
\hline Comprimento & $\boldsymbol{I}$ & $1,00 \mathrm{~m}$ \\
\hline Módulo de Elasticidade & $\boldsymbol{E}$ & $694467,099376 \mathrm{Mpa}$ \\
\hline
\end{tabular}

Fonte: (autoria própria).

A partir das especificações acima e em uso da modelagem teórica e computacional obteve-se os seguintes resultados para ambas as vigas.

\section{RESULTADOS}

$\mathrm{Na}$ implementação computacional utilizou-se a equação 2 com os dados das tabelas 1, 2 e 3 de modo que se pôde representar os quatro primeiros modos de vibração da viga engastada livre e bi engastada, sendo exibidos nos gráficos a seguir. 
Gráfico $1.1^{\circ}$ modo de vibração da viga engastada livre

Fonte: (autoria própria).

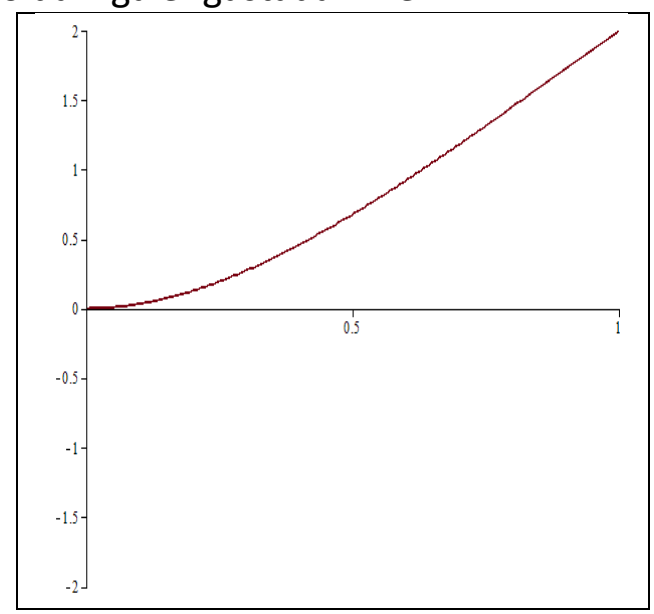

Gráfico 2. $2^{\circ}$ modo de vibração da viga engastada livre

Fonte: (autoria própria).

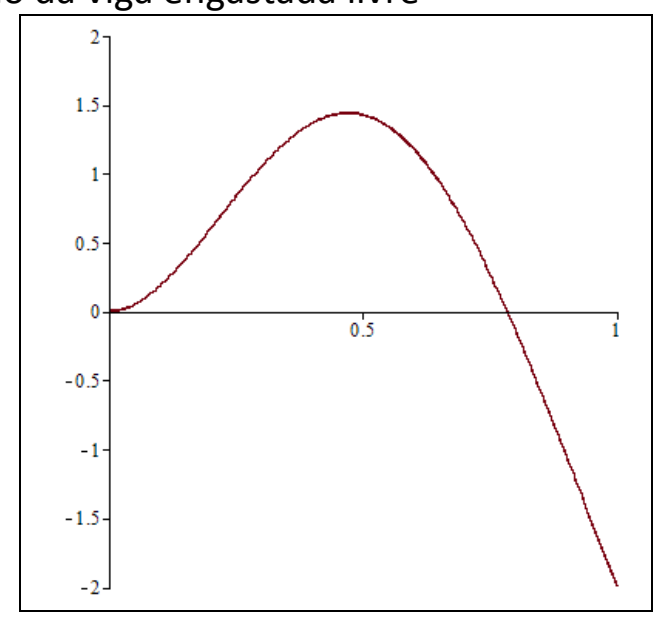

Gráfico 3. $3^{\circ}$ modo de vibração da viga engastada livre

Fonte: (autoria própria).

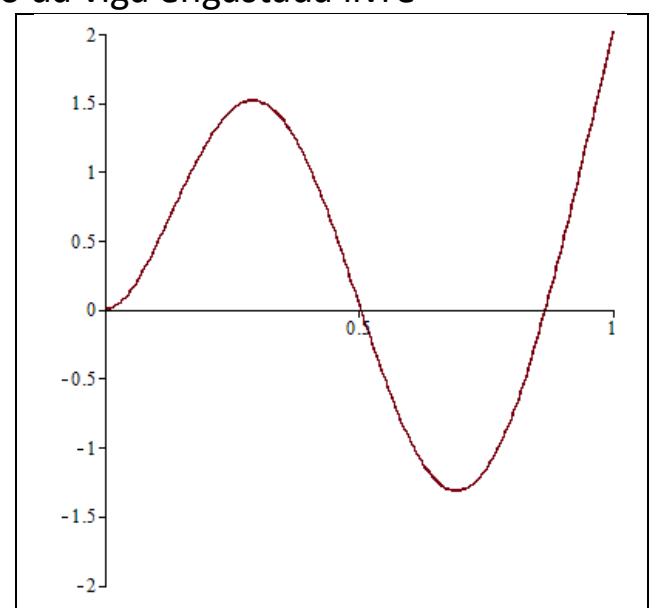


Gráfico $4.4^{\circ}$ modo de vibração da viga engastada livre

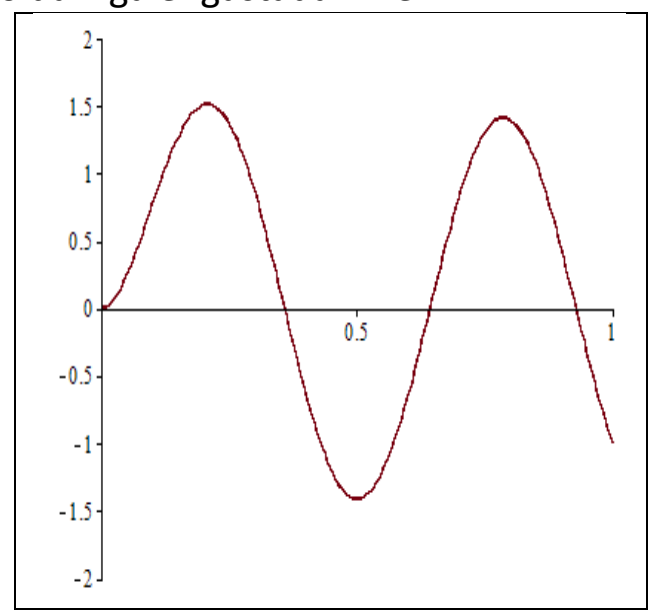

Fonte: (autoria própria).

Gráfico 5. $1^{\circ}$ modo de vibração da viga bi engastada.

Fonte: (autoria própria).

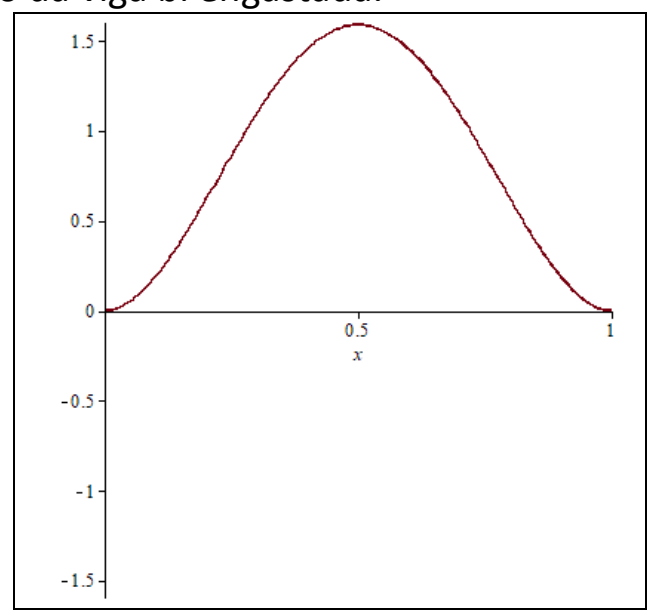

Gráfico 6. $2^{\circ}$ modo de vibração da viga bi engastada.

Fonte: (autoria própria).

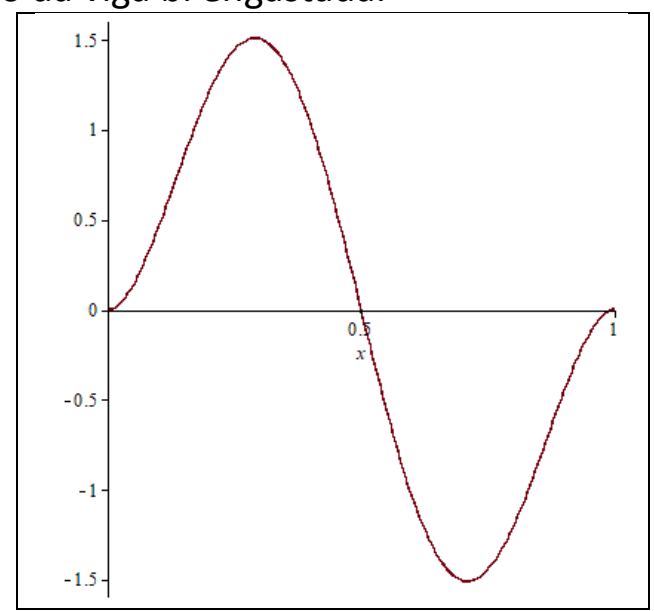


Gráfico 7. $3^{\circ}$ modo de vibração da viga bi engastada.

Fonte: (autoria própria).

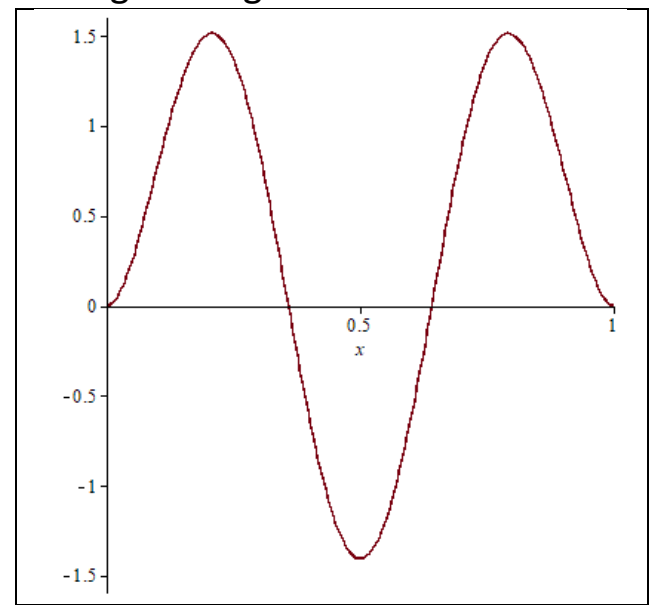

Gráfico 8. $4^{\circ}$ modo de vibração da viga bi engastada.

Fonte: (autoria própria).

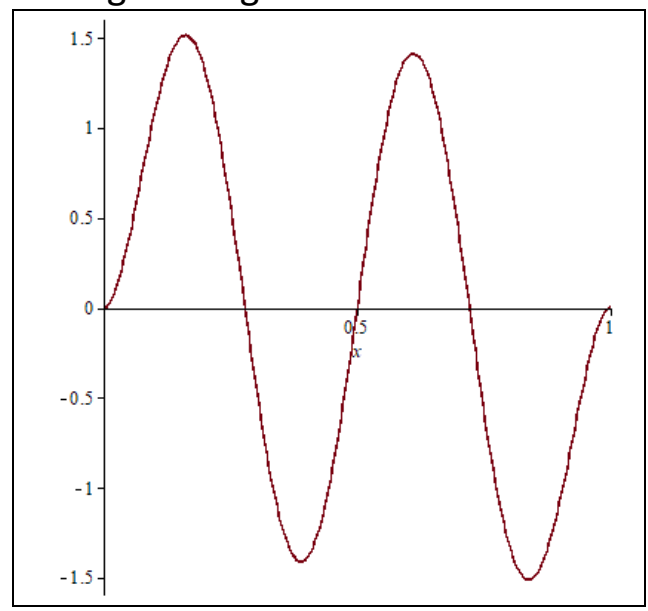

Os gráficos anteriores representam respectivamente as formas dos quatro primeiros modos de vibração para ambas as vigas. Onde 0 eixo $x$ de cada gráfico representa o comprimento em metros de cada viga e o eixo y representa a amplitude da vibração em $10^{-3} \mathrm{~m}$.

Os valores das frequências naturais de vibração para cada modo de cada viga foram obtidos através da equação abaixo, segundo Young e Felgar (1949):

$$
\omega_{i}=\beta_{i} \sqrt{\frac{E . l}{\rho}}
$$

Onde, $i$ representa o $i$-ésimo modo de vibração.

Assim, para a viga engastada livre obteve-se as seguintes frequências naturais de vibração. 
Tabela 4. Frequências naturais de vibração de uma viga engastada livre.

Fonte: (autoria própria)

\begin{tabular}{c|c|c}
\hline $\begin{array}{c}\text { Modo } \\
(\boldsymbol{n})\end{array}$ & $\begin{array}{c}\text { Frequências } \\
\text { naturais } \\
\text { (rad/s) }\end{array}$ & $\begin{array}{c}\text { Frequências } \\
\text { Naturais }(\mathbf{H z})\end{array}$ \\
\hline $\mathbf{1}^{\circ}$ & 123,74105 & 19,69400 \\
\hline $\mathbf{2}^{\circ}$ & 309,76511 & 49,30065 \\
\hline $\mathbf{3}^{\circ}$ & 518,34513 & 82,49719 \\
\hline $\mathbf{4}^{\circ}$ & 725,6119 & 115.48473 \\
\hline
\end{tabular}

$\mathrm{Na}$ tabela a seguir tem-se obtido as frequências naturais de vibração para a viga bi engastada.

Tabela 5. Frequências naturais de vibração de uma viga bi engastada.

Fonte: (autoria própria).

\begin{tabular}{c|c|c}
\hline $\begin{array}{c}\text { Modo } \\
(\boldsymbol{n})\end{array}$ & $\begin{array}{c}\text { Frequências } \\
\text { naturais } \\
\text { (rad/s) }\end{array}$ & $\begin{array}{c}\text { Frequências } \\
\text { Naturais }(\mathbf{H z})\end{array}$ \\
\hline $\mathbf{1}^{\circ}$ & 312,14079 & 49,67875 \\
\hline $\mathbf{2}^{\circ}$ & 518,23957 & 82,48039 \\
\hline $\mathbf{3}^{\circ}$ & 725,61855 & 115,48578 \\
\hline $\mathbf{4}^{\circ}$ & 932,93156 & 148,48067 \\
\hline
\end{tabular}

Vale lembrar que na implementação computacional foram considerados a massa específica, módulo de elasticidade, momento de inércia e consequentemente a geometria da viga.

\section{DISCUSSÃO}

Faz-se importante conhecer as frequências naturais de vibração das estruturas em sua mais variadas condições de contonor, propriedades e vínculos, pois sabendo-se que há a possibilidade da frequência da fonte externa e da viga serem iguais de modo que ocorra a ressonância, absorção de energia de uma fonte externa quando esta está na mesma frequência do receptor. Assim, caso as frequências sejam iguais a estrutura absorverá energia da fonte e como resultado terá sua amplitude aumentada podendo levar a estrutura à ruina ou colapso total.

Este fenômento tem como propriedade conduzir a oscilação da viga ter amplitudes cada vez maiores até o estado crítico da mesma, provocando fissuras, trincas, rupturas e danos irreversíveis a estrutura.

Ressaltar que no decorrer do projeto de uma estrutura, o engenheiro deve realizar os devidos estudos das frequências naturais de vibração de seu projeto. Deste modo, possíveis fontes externas de vibração não serão responsáveis, como sendo fontes de excitação das frequências naturais de vibração do sistema e, consequentemente, no aumento da amplitude de vibração e danos ou colapsos á estruturais.

\section{CONSIDERAÇÕES FINAIS}

Neste trabalho apresentou-se um estudo comparativo da dinâmica de caso entre duas vigas com mesmas propriedades dimensionais e de composição, afim de dar continuidade nos trabalhos de dinâmica na faculdade de Engenharia de Presidente Prudente - SP.

Ao encontrar os modos de vibração de ambos os modelos propostos e suas 
respectivas frequências naturais de vibrações ao empregar o método dos modos assumidos utilizando conceitos da mecânica analítica para descrever os movimentos flexíveis da estrutura.

Assim, as frequências de vibração foram obtidas a partir da função que descreve o movimento oscilatório das vigas segundo a função de forma presente no método dos modos assumidos.

Notadamente as representações gráficas dos quatro primeiros modos de vibração e suas respectivas frequências para a proposta de viga engastada livre como sendo, para cada modo em sequência, 19,7 $\mathrm{Hz}, 49,3 \mathrm{~Hz}, 82,5 \mathrm{~Hz}$ e $115,5 \mathrm{~Hz}$. Para os modos da viga bi engastada tem-se $49,7 \mathrm{~Hz}, 82,5 \mathrm{~Hz}$, $115,5 \mathrm{~Hz}$ e $148,5 \mathrm{~Hz}$.

Tendo em vista que as frequências são únicas para cada condições de contorno e tipo de material conclui-se que cada caso deve ser tratado independentemente mesmo que as propriedades físicas sejam as mesmas das estruturas.

\section{REFERÊNCIAS}

CAMPOS, C. F. S. de; et al. Análise computacional dos modos Ed frequências naturais de vibração de uma viga engastadalivre. Colloquium Exactarum, v. 6, n.1, p.114 - 127, jan/jun. 2014,. DOI: 10.5747/ce.2014.v06.n1.e072.

https://doi.org/10.5747/ce.2014.v06.n1.e072

CAMPOS, C. F. S. Dinâmica e controle de movimento de corpo rígido de um manipulador robótico rígido flexível. Dissertação (Mestrado) - INPE-12308TDI/988. INPE, 2001. 107p.

JUNKINS, J. L.; Kim, Y. Introduction to dynamics and control of flexible structures. Washington: American Institute of Aeronautics and Astronautics, 1993. 452p. https://doi.org/10.2514/4.862076

MEIROVITCH, L. Elements of Vibration Analysis. Tokio: McGraw-Hill, 1975. 495p.
MEIROVITCH, L. Analytical Methods in Vibrations. London: The Macmillan Company, 1967. 555p.

MEIROVITCH, L. Computational Methods in Structural Dynamics. Maryland: Sijthoff \& Noordhoff International Publishers, 1980. 439p.

MENDES, P.; Oliveira, S. Análise Dinâmica de Estruturas: utilização integrada de modelos de identificação modal e modelos de elementos finitos. Lisboa: Laboratório Nacional de Engenharia Civil, 2008.

RODRIGUES, R. O. Análise dinâmica bidimensional não-linear física e geométrica de treliças de aço e pórticos de concreto armado. Tese (Doutorado) - Escola de Engenharia de São Carlos - Universidade de São Paulo. São Carlos. 1997. 275p.

TIMOSHENKO, S., Goodier, J. N. Theory of Elasticity. Tokio: McGraw-Hill Book Company, 1934. 506p.

THOMSON, W. T. Theory of Vibration with Applications. New Jersey: Prentice Hall, 1981. 493p.

WAIDEMAN, L. Análise dinâmica de placas delgadas utilizando elementos finitos triangulares e retangulares. 2004. 153f. Dissertação (Mestrado em Engenharia Civil) Faculdade de Engenharia, Universidade Estadual Paulista, Ilha Solteira, 2004.

YOUNG, D.; FELGAR, P. Jr. Tables of Characteristic Functions Representing Normal Modes of Vibration of a Beam. The University of Texas Publication. N 4913. Texas, 1949. 